\title{
Chaotic Quivering of Micron-Scaled On-Chip Resonators Excited by Centrifugal Optical Pressure
}

\author{
Tal Carmon, M. C. Cross, and Kerry J. Vahala \\ California Institute of Technology, 1200 E. California Blvd, Pasadena, California 91125 USA
}

(Received 26 September 2006; published 19 April 2007)

\begin{abstract}
Opto-mechanical chaotic oscillation of an on-chip resonator is excited by the radiation-pressure nonlinearity. Continuous optical input, with no external feedback or modulation, excites chaotic vibrations in very different geometries of the cavity (both tori and spheres) and shows that opto-mechanical chaotic oscillations are an intrinsic property of optical microcavities. Measured phenomena include period doubling, a spectral continuum, aperiodic oscillations, and complex trajectories. The rate of exponential divergence from a perturbed initial condition (Lyapunov exponent) is calculated. Continuous improvements in cavities mean that such chaotic oscillations can be expected in the future with many other platforms, geometries, and frequency spans.
\end{abstract}

DOI: 10.1103/PhysRevLett.98.167203

PACS numbers: $85.85 .+\mathrm{j}, 42.50 . \mathrm{Vk}, 42.65 .-\mathrm{k}$

Radiation pressure (RP) can push the boundaries of an optical cavity to change its optical path [1-4]. In the past, the resulting coupling between optical and mechanical motions was shown to induce a hysteretic response [1] that characterizes a bistable regime. In this regime, the system can settle in one of two stable equilibria wherein mechanical inflation of the cavity is balanced by the resulting attenuation of the intracavity circulating optical power. Reducing the loss has been recently shown to opto-excite periodic vibrations [5-7] in which mechanical gain (via Doppler shift of the intracavity light) overcomes the mechanical loss. It was shown [5] that at higher powers, a transition to erratic behavior is observed. However, so far there has not been an experimental (or theoretical) study of this regime.

Here we complete the set of dynamic regimes (bistable, periodic, and chaotic) by experimentally demonstrating how a continuous wave $(\mathrm{CW})$ optical input excites the chaotic regime. The chaotic behavior is demonstrated by the experimental observation of period doubling, a spectrum that turns from a set of discrete lines into a continuum, and a complex phase-space trajectory. The sensitivity of the system dynamics for infinitesimal changes in initial conditions is also shown.

Unlike other systems possessing optical chaos by nonlinearities originating from interaction of light with matter [8] (e.g., intensity dependant index of refraction or absorption), the interaction in here is between light and structure, through boundary dynamics, resulting in inertia for the optical pathlength via direct coupling to the inertia of the mechanical mode. Another result is that the time constant of chaos scales as the resonator acoustical time constant [size/(velocity of sound)] and not with time constants at the molecular level. Effects like the continuum spectra observed here extend phenomena previously observed at the molecular scale to ones originating in our work from structural-level vibrations of the photonic device itself. The fact that the sound wave extends out of the cavity through its support (see rendering of the calculated mode in Fig. 1(a)) suggests future possibilities for acoustical coupling to nearby resonators through the wafer [9] or for coupling such sound waves to a distant resonator via electro-mechanical transduction. Another coupling option is via the optical link through the fiber. Also different is that while some chaotic optical systems necessitate periodic perturbation $[10,11]$ or an external delay line feedback [12] in order for chaos to develop, chaos here evolves from a continuous-in-time input, necessitating no periodic perturbation, external feedback, modulation, or delay; and by this, showing that chaotic vibration here is an intrinsic cavity property. The opto-mechanical system described here bridges between the (chaotic) mechanical- and optical-resonator and is of interest for various communities investigating micro-electro-mechanical systems (MEMS), nonlinear optics, and micro-cavities.

The simple experimental setup (Fig. 1(a)) consists of a $\mathrm{CW}$ optical-input $(\lambda=1.5 \mu \mathrm{m})$ that is evanescently coupled to and from a spherical cavity (made using a technique similar to the one described in [13]) through a tapered fiber $[14,15]$. The radiation pressure of the intracavity light causes the cavity to inflate, forcing the optical resonance wavelength to expand proportionally. This resonance drift (from the pump wavelength) turns off the circulating intracavity light. With no light inside to maintain the mechanical flex, the cavity deflates back towards mechanical equilibrium and is charged again with light. The cycle repeats itself then perpetually but not necessarily periodically. This qualitative description will be cast into a set of dynamical equations; however, it is important to already note that the radiation-pressure induced chaotic vibration is not limited to special geometry of the resonator, as will be shown by repeating the experiments in very different geometries.

At low input intensity, the optical power spectrum (Fig. 1(b)) emitted from the spheroid resonator is periodically modulated at $0.538 \mathrm{GHz}$ by the oscillation of a 


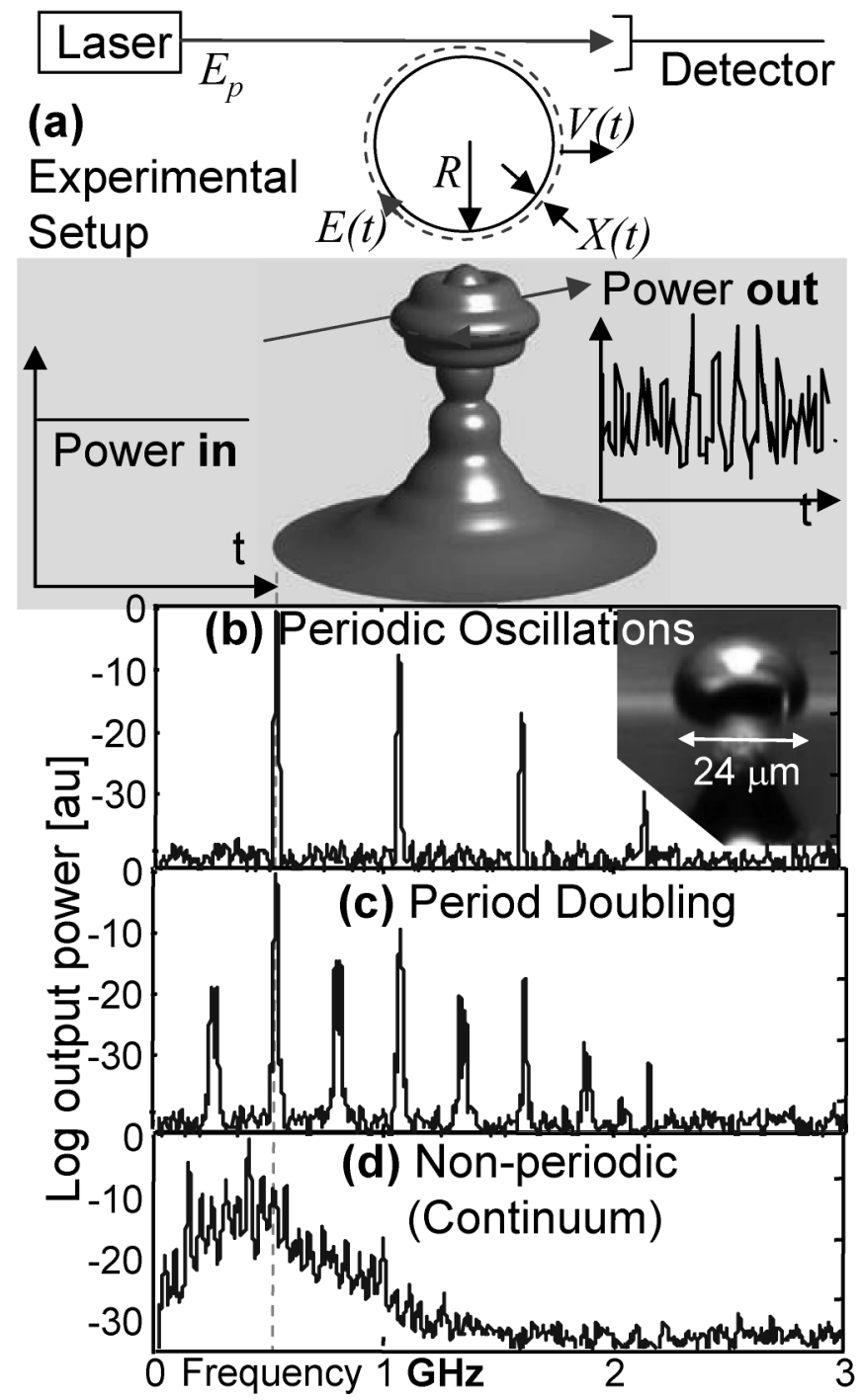

FIG. 1. Experimental setup (a) and results (b)-(d): (a) Continuous optical power is fiber coupled to an on-chip microresonator in which the centrifugal radiation pressure excites mechanical vibration. The calculated vibrational mode for the spherical cavity used here is presented in a rendering where the deformation describes the cavity shape at maximum mechanical amplitude. The deformation is exaggerated in the figure. The experiment is done at room temperature and pressure. $R$ signifies the cavity radius. (b) Oscillation in spherical cavity starts periodic (c), doubles its period (d), and then turns aperiodic (continuous spectra) as input power increases. Inset in (b) is a micrograph of the spherical resonator used in this experiment. Panels b, c, and d were taken at pump power $\left(\left|E_{p}\right|^{2}\right)$ of 66,79 , and $83 \mathrm{~mW}$. The sphere radius is $12 \mu \mathrm{m}$, its optical $Q$ is $7 \times$ $10^{6}$, and its mechanical $Q$ is 112 .

mechanical mode (the calculated mechanical mode is shown in Fig. 1(a)); higher harmonics in this optical spectra (Fig. 1(b)) indicate that the system is highly nonlinear. Highly nonlinear dynamical systems often become chaotic and the route from regular to chaotic behavior is generally characterized by period doubling $[16,17]$. Such period doubling (frequency halving) is observed in
Fig. 1(c); it is important to note that this observed frequency halving is not due to a lower order mechanical mode as calculations show that no mechanical mode with such a frequency exists. As for higher-order cascaded period doubling, many such doublings are observed when a high-resolution scan is performed as shown in the supplementary movies [18]. At yet higher input-power levels, the power spectrum turns from a discrete set of equally-separated lines into a continuum (Fig. 1(d)). A description of spectrum evolving to continuum (as described in Fig. 1(b)-1(d)) is given as supplementary movies [18].

We emphasize that the continuum in Fig. 1(d) is not generated from a spectrally-wide pulsed input; the frequency continuum here is excited from a continuous-intime $(\mathrm{CW})$ source which is very narrow in the frequency domain (300 kHz optical bandwidth). Furthermore, in all experiments here, chaotic oscillations lasted as long as we kept our CW input source on (many hours).

In the next experiment, the spherical oscillator is replaced with a toroidal oscillator (Fig. 2) to illustrate the operation in a different frequency regime as well as to show that the phenomena are not limited to a specific oscillator geometry. Toroids are more flexible than spheres and oscillate mechanically at lower frequencies. The spectral evolution for the toroid is the same as for the sphere except for the appearance of a period-four cycle (i.e., two period-doubling bifurcations). The unpredictable character of the measured complex trajectory (Fig. 2(c)) is the result of sensitivity for small changes in initial condition and not from stochastic ("coin flip") terms in the governing equations. Another general characteristic of the perioddoubling route (shown experimentally in Fig. 2(c)) is that while the period 2 and 4 cycles are broadened and washed into a continuum [19,20], the fundamentalfrequency component, as well as its high harmonics, still survive (Fig. 2(c)).

Sensitivity to initial conditions is a defining property of chaos. To investigate this separation of trajectories for identical systems with infinitesimally close initial conditions, we calculate the Lyapunov exponent in a theoretical model of the experimental system [21]. We first bring the equations that govern [5] the cavity mechanical deformation, $X_{(t)}$, and the slowly varying electric field of the circulating optical mode, $E_{(t)}=E_{\mathrm{re}(t)}+i E_{\operatorname{im}(t)}$ (see the variables sketched in Fig. 1(a)) to an autonomous form where no time appears (explicitly) on the right hand side and no imaginary numbers are involved:

$$
\begin{aligned}
\dot{X}_{(t)} & =V_{(t)}, \\
\dot{V}_{(t)} & =-a V_{(t)}-b X_{(t)}+c\left(E_{\mathrm{re}(t)}{ }^{2}+E_{\mathrm{im}(t)}{ }^{2}\right), \\
\dot{E}_{\mathrm{re}(t)} & =\left(e-f X_{(t)}\right) E_{\mathrm{im}(t)}-d E_{\mathrm{re}(t)}, \\
\dot{E}_{\mathrm{im}(t)} & =g E_{p}-\left(e-f X_{(t)}\right) E_{\mathrm{re}(t)}-d E_{\mathrm{im}(t)}
\end{aligned}
$$

The first two expressions in Eq. (1) characterize a mechanical harmonic oscillator, and the last two expressions de- 


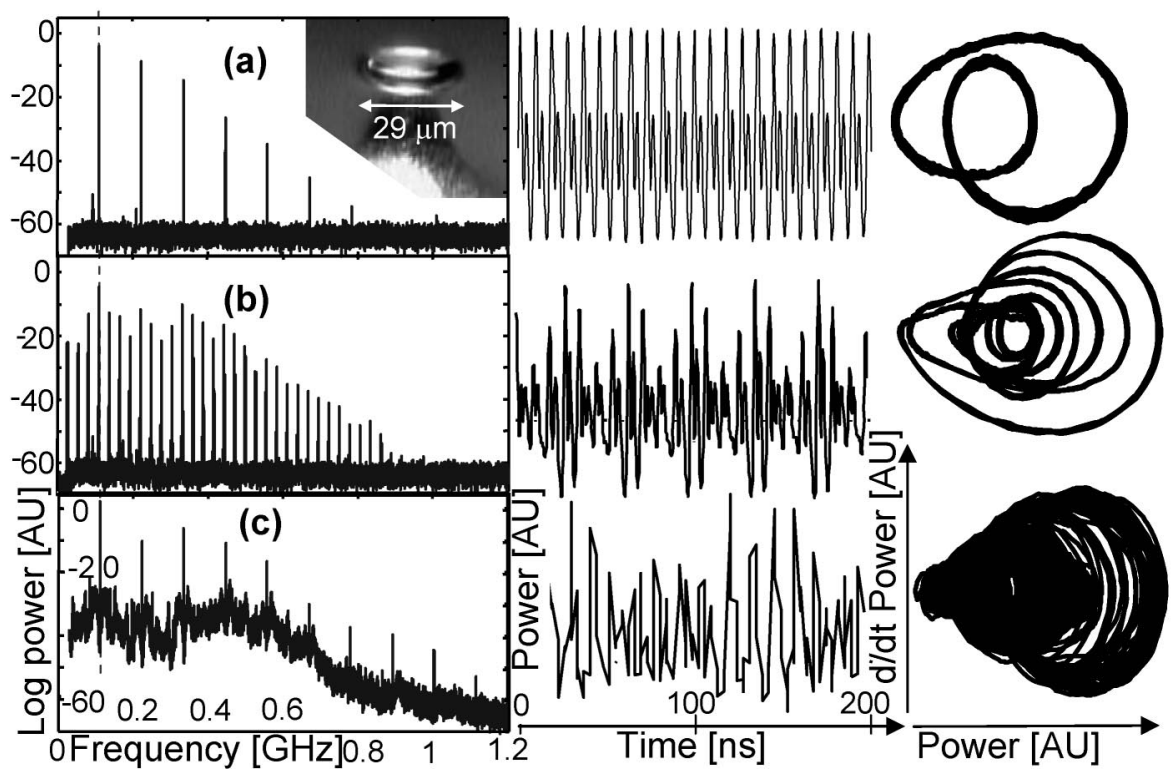

FIG. 2. Experimental results: As the input power increases, the periodic oscillation (a) of a toroidal microcavity doubles its period twice (b) and then turns aperiodic (c). Oscillation of the output power is measured in the frequency domain as well as in the temporal domain. Phase-space plots of the first derivative of the measured output power in time versus the measured output power are also shown. The inset in (a) shows a toroid like the one investigated here. Panels a, b, and c were taken at pump power $\left(\left|E_{p}\right|^{2}\right)$ of 11,21 , and $28.7 \mathrm{~mW}$. The mechanical $Q$ is 250 , and the optical $Q$ is $10^{7}$. Major and minor toroid radii are 14.5 and $3 \mu \mathrm{m}$.

scribe the rate of change of the intracavity optical field when pumped by a continuous power $\left|E_{p}\right|^{2}$ and deformed to inflate by $X_{(t)}$ (see constants $a-g$ in Ref. [22]). These equations are coupled as the mechanical oscillator is driven by the optical power via the $c\left(E_{\mathrm{re}(t)}^{2}+E_{\mathrm{im}(t)}^{2}\right)$ term, and the intracavity field (last two equations) depends on the mechanical deformation $\left(X_{(t)}\right)$ through cavity detuning. It is convenient to use the MKS system of units with $E$ taken

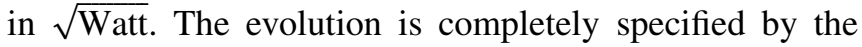
instantaneous values (of $V_{(t)}, X_{(t)}$, and $E_{(t)}$ ), and there are no stochastic terms in the equations. The equations are nonlinear through the quadratic terms in the second equation and the mixed terms $\left(E_{(t)} X_{(t)}\right)$ in the last two equations. Lastly, dissipation, included via the "diagonal" terms ( $a$ for mechanical dissipation and $d$ for optical dissipation), implies that the solution is bounded and that phase space will converge to a point upon turning off the input power $\left(\left|E_{p}\right|^{2}\right)$. An analytical solution for Eq. (1) was recently demonstrated in the subchaotic regime [23]. However, the aperiodic behavior (Fig. 2(c)) implies that a numerical solution is likely to be helpful here.

The evolution of a perturbation $\vec{\varepsilon}=(\delta X, \delta V$, $\left.\delta E_{\mathrm{re}}, \delta E_{\mathrm{im}}\right)$ is derived by linearizing Eq. (1) to become

$$
\begin{aligned}
\delta \dot{X}_{(t)}= & \delta V_{(t)} \\
\delta \dot{V}_{(t)}= & -a \delta V_{(t)}-b \delta X_{(t)}+2 c\left(E_{\mathrm{re}(t)} \delta E_{\mathrm{re}(t)}\right. \\
& \left.+E_{\mathrm{im}(t)} \delta E_{\mathrm{im}(t)}\right) \\
\delta \dot{E}_{\mathrm{re}(t)}= & \left(e-f X_{(t)}\right) \delta E_{\mathrm{im}(t)}-f \delta X_{(t)} E_{\mathrm{im}(t)}-d \delta E_{\mathrm{re}(t)}, \\
\delta \dot{E}_{\mathrm{im}(t)}= & \left(-e+f X_{(t)}\right) \delta E_{\mathrm{re}(t)}+f \delta X_{(t)} E_{\mathrm{re}(t)}-d \delta E_{\mathrm{im}(t)} .
\end{aligned}
$$

Solving the above 8 differential equations numerically by using the predictor-corrector Adams's method reveals a low input-power subchaotic regime in which the dynamics is periodic (calculated in Fig. 3(a) RHS and observed experimentally in Fig. 1(b) and 2(a)). The flat evolution of $\log [\delta$ output power $]$ in Fig. 3(a) indicates that in this subchaotic regime, the trajectories of two systems with infinitesimally different initial condition will not diverge. This dynamic is referred to as a limit-cycle, to describe an attracting set to which trajectories converge and upon which trajectories are periodic.

At higher optical-input power, a transition to an aperiodic oscillation appears, and the calculated system behavior (Fig. 3(b) RHS) is as measured in Fig. 1(d) and 2(c). In this input power, the calculated exponential divergence of $\delta$ output power (Fig. 3(b) LHS) indicates a different regime in which initially nearby points in phase space evolve into completely different states separating as shown in Fig. 3(b) (LHS). The logarithmic slope defines the Lyapunov exponent and quantifies the chaotic degree of the system and its sensitivity to slight changes in the initial conditions. The behavior in the chaotic regime is characterized (among other things) by the structure of the phasespace trajectory. In this regard, the calculated phase-space trajectory in Fig. 3(b) exhibits structure similar to the one measured in Fig. 2(c) as evident by the empty regions at the center and on the right, and by the stick out on its left. Also in the subchaotic regime, the calculated phase space (Fig. 3(a)) is similar to the measured one (Fig. 2(a)). In this Letter, we have made the first experimental study of opto-excited chaotic oscillations induced by radiation pressure. Using a continuous optical input as well as repeating 

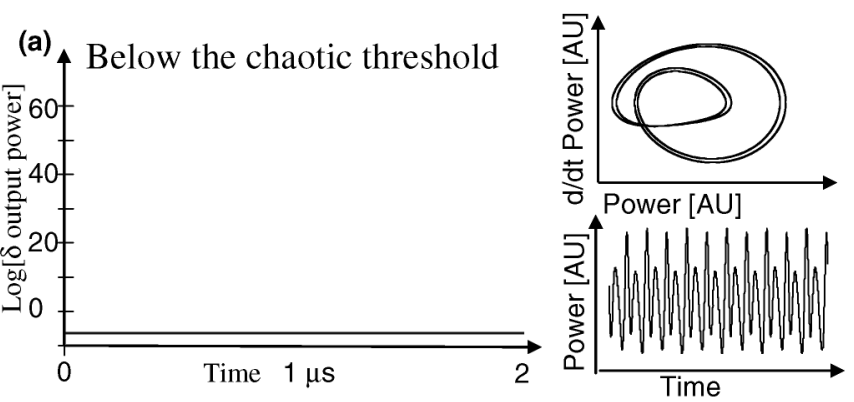

(b) $\uparrow$ Above the chaotic threshold

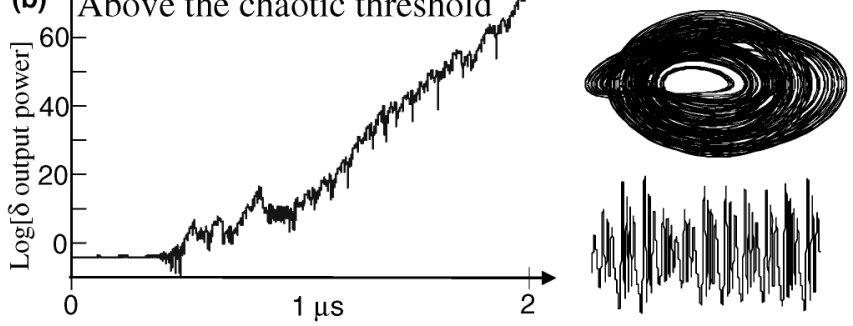

FIG. 3. Theoretical calculation. (a): $\log [\delta$ output power $]$ vs time for low input power exhibits a flat evolution. Parameters are the same as those of the experiment in Fig. 2 and were measured to be $a=1.4 \times 10^{6}, b=1.2 \times 10^{17}, c=9779, d=$ $1.2 \times 10^{8}, e=1.3 \times 10^{8}, f=1.1 \times 10^{20}, g=2.2 \times 10^{10}$ all in MKS. Mechanical- and optical-damping is weak (b), increasing the input power (to be the same as in experiment with details in Fig. 2(c)) causes exponential divergence of $\delta$ output power . Insets are the calculated evolution in phase space and time exhibiting similar behavior to the one measured in Fig. 2.

the experiments in different geometries (tori and spheres) shows that this chaotic vibration is an intrinsic cavity property requiring no external feedback or external modulation and is not limited to a specific geometry. Along this line, it seems likely that the regime of chaos is also obtainable in cantilever [23] and other opto-mechanical cavities. Different from optical chaos in which laser action is necessary [24], the platform here is laser independent. Yet, integration of a laser in a device as in Fig. 1 is possible [25], allowing further degrees of freedom for future research. Our platform allows freedom in choosing different optical wavelengths $(260-2000 \mathrm{~nm})$, changing the coupling constant, selecting the geometry and selecting highorder mechanical modes to allow vibrational frequencies from $\mathrm{MHz}$ to above $\mathrm{GHz}$. We can also change the detuning between the optical wavelength of the source and the optical resonance wavelength of the cavity at mechanical equilibrium. Another possibility is cascading many such opto-mechanical resonators either optically via the fiber or acoustically via the silicon wafer [9]. Additionally, by changing cavity size and shape, chaotic oscillation can be engineered to span from $\mathrm{MHz}$ to $\mathrm{GHz}$ vibration rates and above [26]. To conclude, there is growing interest in the manifestation of radiation pressure as an opto-mechanical nonlinearity for various applications as suggested theoretically in Refs. [27-29]. Taking into account the continued trend of miniaturization and loss reduction in optical mi- crocavities of all forms, we believe that opto-excited chaotic vibration will soon be relevant to more and more devices over many platforms operating at yet higher frequencies.

[1] A. Dorsel et al., Phys. Rev. Lett. 51, 1550 (1983).

[2] V. B. Braginsky et al., Phys. Lett. A 179, 244 (1993).

[3] P. F. Cohadon et al., Phys. Rev. Lett. 83, 3174 (1999).

[4] C. M. Mow-Lowry et al., Phys. Rev. Lett. 92, 161102 (2004).

[5] T. Carmon et al., Phys. Rev. Lett. 94, 223902 (2005).

[6] H. Rokhsari et al., Opt. Express 13, 5293 (2005).

[7] T. J. Kippenberg et al., Phys. Rev. Lett. 95, 033901 (2005).

[8] K. Ikeda et al., Phys. Rev. Lett. 45, 709 (1980).

[9] M. Sato et al., Chaos 13, 702 (2003).

[10] P. Meystre et al., J. Opt. Soc. Am. B 2, 1830 (1985).

[11] W. Klische et al., Opt. Lett. 9, 561 (1984).

[12] H. M. Gibbs et al., Phys. Rev. Lett. 46, 474 (1981).

[13] L. Yang et al., Appl. Phys. Lett. 83, 825 (2003).

[14] M. Cai et al., Phys. Rev. Lett. 85, 74 (2000).

[15] S. M. Spillane et al., Phys. Rev. Lett. 91, 043902 (2003).

[16] M. J. Feigenbaum, J. Stat. Phys. 21, 669 (1979).

[17] H.D. I. Abarbanel et al., Introduction to Nonlinear Dynamics for Physicists (World Scientific, Singapore; New Jersey, 1993), pp. viii.

[18] See EPAPS Document No. E-PRLTAO-98-069716. Supplementary movie 1: Route to chaos. As input power increases, the spectrum of the out-coupled light changes from a set of discrete lines to continuum. A few perioddoubling events (frequency halving) are seen during this evolution. Parameters as in Fig. 1. Supplementary movie 2: Multiple events of period doublings are seen when scan is performed at higher resolution. For more information on EPAPS, see http://www.aip.org/pubservs/epaps.html.

[19] B. A. Huberman and A. B. Zisook, Phys. Rev. Lett. 46, 626 (1981).

[20] A. Wolf and J. Swift, Phys. Lett. A 83, 184 (1981).

[21] V. I. Oseledec, Trans Mosc. Mat. Soc. 19, 179 (1968).

[22] $a=\omega_{a} / Q_{a}, b=\omega_{a}{ }^{2}, c=n /\left(c_{0} \rho \pi r^{2} R\right), e=\omega_{0}-\bar{\omega}_{0}$, $d=\alpha c_{0} / n, f=\omega_{0}^{2} n /\left(c_{0} N\right), g=\sqrt{\alpha c_{0} / n \tau}$, where $c_{0}$ is the vacuum velocity of light, $n$ is the refractive index, $\alpha$ is the absorption coefficient in cavity, $\rho$ is the material density, $\omega$ and $Q$ are frequency and quality factor with subscript " $a$ " for mechanical and " $o$ " for optical. $\bar{\omega}_{0}$ is the optical resonance at mechanical equilibrium. $R$ and $r$ are the major and minor toroid radii. $N$ is the number of optical wavelengths resonating along the circumference, and $\tau$ is the time it takes the light to accomplish one round trip in cavity. Parameters were calculated from experimental measurement.

[23] F. Marquardt et al., Phys. Rev. Lett. 96, 103901 (2006).

[24] C. O. Weiss and J. Brock, Phys. Rev. Lett. 57, 2804 (1986).

[25] L. Yang et al., Appl. Phys. Lett. 86, 091114 (2005).

[26] T. Carmon and K. J. Vahala, Phys. Rev. Lett. 98, 123901 (2007).

[27] W. Marshall et al., Phys. Rev. Lett. 91, 130401 (2003).

[28] S. Mancini et al., Phys. Rev. Lett. 90, 137901 (2003).

[29] M. L. Povinelli, Opt. Lett. 30, 3042 (2005). 